\title{
SBI and EHR: understanding, adoption, and implementation in family medicine clinics
}

\author{
Roger Zoorob ${ }^{1 *}$, Sandra J Gonzalez ${ }^{1}$, Heather Snell ${ }^{2}$, Heather O'Hara ${ }^{2}$, Mohamad Sidani ${ }^{1}$ \\ From INEBRIA 12th Congress, \\ Atlanda, GA, USA. 24-25 September 2015
}

\section{Background}

Alcohol screening and brief intervention (SBI) programs have been shown to be effective in reducing risky alcohol consumption among primary care patients [1-3]. Although various implementation protocols exist, it can be difficult to launch and sustain SBI programs. A number of barriers exist, including those related to clinical workflow, the intake process, and the incorporation of protocols into electronic health records (EHR)[4-6].

This study aims to present challenges and potential solutions to incorporating SBI as a standard of care into an existing EHR of a family medicine system.

\section{Material and methods}

An SBI program was piloted in two underserved family medicine clinical teaching practices. Physicians, residents, nurses, medical assistants and patient service representatives (PSR's) were trained on the protocol for adopting this practice into daily clinic work. Through this implementation, an EHR template was created to complement the workflow.

\section{Results}

Utilization of the screening tool provided a mechanism to better assess risky drinking within a regular patient encounter. High rates of completion were appreciated throughout the grant period prior to leadership changes. The EHR template facilitated the intake process, the clinical encounter, and provided a mechanism for billing.

\section{Conclusions}

The incorporation of a dedicated EHR template may mitigate providers' concerns about time constraints and

\footnotetext{
* Correspondence: Roger.Zoorob@bcm.edu

${ }^{1}$ Family and Community Medicine, Baylor College of Medicine, Houston, USA Full list of author information is available at the end of the article
}

establishes a more effective mechanism for billing for the service.

Strong support from organizational leadership and the use of clinic champions were shown to positively contribute to the success of the SBI programs in this health system by addressing common barriers to implementation.

\section{Acknowledgements}

We would like to thank the Fetal Alcohol Syndrome Prevention Team, National Center on Birth Defects and Developmental Disabilities, Centers for Disease Control and Prevention for their support of this project.

\section{Authors' details}

${ }^{1}$ Family and Community Medicine, Baylor College of Medicine, Houston, USA. ${ }^{2}$ Family and Community Medicine, Meharry Medical College, Nashville, USA.

\section{Published: 24 September 2015}

\section{References}

1. Zoorob $R$, et al: Screening and brief intervention for risky alcohol use. Curr Probl Pediatr Adolesc Health Care 2014, 44(4):82-7.

2. Jonas $D E$, et al: Behavioral counseling after screening for alcohol misuse in primary care: a systematic review and meta-analysis for the US Preventive Services Task Force. Annals of internal medicine 2012, 157(9):645-654.

3. O'Donnell $A$, et al: The impact of brief alcohol interventions in primary healthcare: a systematic review of reviews. Alcohol Alcohol 2014, 49(1):66-78.

4. Williams EC, et al: Strategies to implement alcohol screening and brief intervention in primary care settings: a structured literature review. Psychol Addict Behav 2011, 25(2):206-14.

5. Amaral MB, Ronzani TM, Souza-Formigoni ML: Process evaluation of the implementation of a screening and brief intervention program for alcohol risk in primary health care: An experience in Brazil. Drug Alcohol Rev 2010, 29(2):162-8.

6. Tai B, Wu LT, Clark HW: Electronic health records: essential tools in integrating substance abuse treatment with primary care. Subst Abuse Rehabil 2012, 3:1-8.

doi:10.1186/1940-0640-10-S2-048

Cite this article as: Zoorob et al: SBI and EHR: understanding, adoption, and implementation in family medicine clinics. Addiction Science \& Clinical Practice 2015 10(Suppl 2):O48. 